\title{
Temporal Discourse Models for Narrative Structure
}

\author{
Inderjeet MANI \\ Department of Linguistics \\ Georgetown University \\ ICC 452 \\ Washington, DC 20057 \\ im5@georgetown.edu
}

\begin{abstract}
Getting a machine to understand human narratives has been a classic challenge for NLP and AI. This paper proposes a new representation for the temporal structure of narratives. The representation is parsimonious, using temporal relations as surrogates for discourse relations. The narrative models, called Temporal Discourse Models, are treestructured, where nodes include abstract events interpreted as pairs of time points and where the dominance relation is expressed by temporal inclusion. Annotation examples and challenges are discussed, along with a report on progress to date in creating annotated corpora.
\end{abstract}

\section{Introduction}

Getting a machine to understand human narratives has been a classic challenge for NLP and AI. Central to all narratives is the notion of time and the unfolding of events. When we understand a story, in addition to understanding other aspects such as plot, characters, goals, etc., we are able to understand the order of happening of events. A given text may have multiple stories; when we understand such a text, we are able to tease apart these distinct stories. Thus, understanding the story from a text involves building a global model of the sequences of events in the text, as well as the structure of nested stories. We refer to such models as Temporal Discourse Models (TDMs).

Currently, while we have informal descriptions of the structure of narratives, e.g., (Bell 1999), we lack a precise understanding of this aspect of discourse. What sorts of structural configurations are observed? What formal characteristics do they have? For syntactic processing of natural languages, we have, arguably, answers to similar questions. However, for discourse, we have hardly begun to ask the questions.

One of the problems here is that most of the information about narrative structure is implicit

\author{
James PUSTEJOVSKY \\ Department of Computer Science \\ Brandeis University \\ Volen 258 \\ Waltham, Massachusetts 02254 \\ jamesp@cs.brandeis.edu
}

in the text. Thus, while linguistic information in the form of tense, aspect, temporal adverbials and discourse markers is often present, people use commonsense knowledge to fill in information. Consider a simple discourse: Yesterday Holly was running a marathon when she twisted her ankle. David had pushed her. Here, aspectual information indicates that the twisting occurred during the running, while tense suggests that the pushing occurs before the twisting. Commonsense knowledge also suggests that the pushing caused the twisting.

We can see that even for interpreting such relatively simple discourses, a system might require a variety of sources of linguistic knowledge, including knowledge of tense, aspect, temporal adverbials, discourse relations, as well as background knowledge. Of course, other inferences are clearly possible, e.g., that the running stopped after the twisting, but when viewed as defaults, these latter inferences seem to be more easily violated. The need for commonsense inferences has motivated computational approaches that are domainspecific, using hand-coded knowledge (e.g., Asher and Lascarides 2003, Hitzeman et al. 1995).

A number of theories have postulated the existence of various discourse relations that relate elements in the text to produce a global model of discourse, e.g., (Mann and Thompson 1988), (Hobbs 1985), (Hovy 1990) and others. In RST (Mann and Thompson 1988), (Marcu 2000), these relations are ultimately between semantic elements corresponding to discourse units that can be simple sentences or clauses as well as entire discourses. In SDRT (Asher and Lascarides 2003), these relations are between representations of propositional content, called Discourse Representation Structures (Kamp and Reyle, 1993).

Despite a considerable amount of very productive research, annotating such discourse relations has proved problematic. This is due to the fact that discourse markers may be absent (i.e., implicit) or ambiguous; but more importantly, because in many cases the precise nature of these discourse relations is unclear. Although (Marcu et 
al. 1999) (Carlson et al. 2001) reported relatively high levels of inter-annotator agreement, this was based on an annotation procedure where the annotators were allowed to iteratively revise the instructions based on joint discussion.

While we appreciate the importance of representing rhetorical relations in order to carry out temporal inferences about event ordering, we believe that there are substantial advantages in isolating the temporal aspects and modeling them separately as TDMs. This greatly simplifies the representation, which we discuss next.

\section{Temporal Discourse Models}

A TDM is a tree-structured syntactic model of global discourse structure, where temporal relations are used as surrogates for discourse relations, and where abstract events corresponding to entire discourses are introduced as nodes in the tree.

We begin by illustrating the basic intuition. Consider discourse (1), from (Webber 1988):

(1) a. John went into the florist shop.

b. He had promised Mary some flowers. c. She said she wouldn't forgive him if he forgot. $d$. So he picked out three red roses.

The discourse structure of (1) can be represented by the tree, T1, shown below.

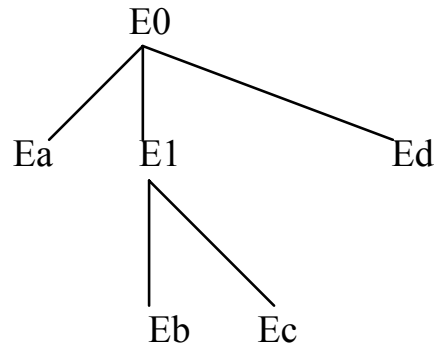

Here E0 has children Ea, E1, and Ed, and $\mathrm{E} 1 \mathrm{has}$ children $\mathrm{Eb}$ and Ec. The nodes with alphabetic subscripts are events mentioned in the text, whereas nodes with numeric subscripts are abstract events, i.e., events that represent abstract discourse objects. A node $\mathrm{X}$ is a child of node $\mathrm{Y}$ iff $\mathrm{X}$ is temporally included in $\mathrm{Y}$. In our scheme, events are represented as pairs of time points. So, E0 is an abstract node representing a top-level story, and E1 is an abstract node representing an embedded story. Note that the mentioned events are ordered left to right in text order for notational convenience, but no temporal ordering is directly represented in the tree. Since the nodes in this representation are at a semantic level, the tree structure is not necessarily isomorphic to a representation at the text level, although $\mathrm{T} 1$ happens to be isomorphic.
In addition to $\mathrm{T} 1$, we also have the temporal ordering constraints $\mathrm{C} 1: \quad\{\mathrm{Eb}<\mathrm{Ec}, \mathrm{Ec}<$ $\mathrm{Ea}, \mathrm{Ea}<\mathrm{Ed}\}$. These are represented separately from the tree. A TDM is thus a pairing of tree structures and temporal constraints. More precisely, a Temporal Discourse Model for a text is a pair $\langle\mathrm{T}, \mathrm{C}\rangle$, where $\mathrm{T}$ is a rooted, unordered, directed tree with nodes $\mathrm{N}=\{\mathrm{E} \cup \mathrm{A}\}$, where $\mathrm{E}$ is the set of events mentioned in the text and $\mathrm{A}$ is a set of abstract events, and a parent-child ordering relation, $\subseteq$ (temporal inclusion). A non-leaf node can be textually mentioned or abstract. Nodes also have a set of atomic-valued features. Note that the tree is temporally unordered left to right. $\mathrm{C}$ is a set of temporal ordering constraints using the ordering relation, < (temporal precedence) as well as (for states, clarified below) 'minimal restrictions' on the above temporal inclusion relation (expressed as $\mathrm{a} \subseteq$ min).

In (1) the embedding nodes E0 and E1 were abstract, but textually mentioned events can also create embeddings, as in (2) (example from (Spejewski 1988)):

(2) a. Edmond made his own Christmas presents this year. b. First he dried a bunch of tomatoes in his oven. $c$. Then he made a booklet of recipes that use dried tomatoes. $d$. He scanned in the recipes from his gourmet magazines. e. He gave these gifts to his family.

$\mathrm{T} 2=$

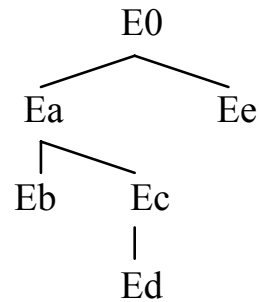

\section{$\mathrm{C} 2=\{\mathrm{Ea}<\mathrm{Ee}, \mathrm{Eb}<\mathrm{Ec}\}$}

Note that the partial ordering $\mathrm{C}$ can be extended using $\mathrm{T}$ and temporal closure axioms (Setzer and Gaizauskas 2001), (Verhagen 2004), so that in the case of $\langle\mathrm{T} 2, \mathrm{C} 2\rangle$, we can infer, for example, that $\mathrm{Eb}<\mathrm{Ed}, \mathrm{Ed}<\mathrm{Ee}$, and so forth.

In representing states, we take a conservative approach to the problems of ramification and change (McCarthy and Hayes 1969). This is the classic problem of recognizing when states (the effects of actions) change as a result of actions. Any tensed stative predicate will be represented as a node in the tree (progressives are here treated as stative). Consider an example like John walked home. He was feeling great. Here we represent the state of feeling great as being minimally a part of the event of walking, without committing to whether it extends before or 
after the event. While this is interpreted as an overloaded temporal inclusion in the TDM tree, a constraint is added to $\mathrm{C}$ indicating that this inclusion is minimal.

This conservative approach results in logical incompleteness, however. For example, given the discourse Max entered the room. He was wearing a black shirt, the system will not know whether the shirt was worn after he entered the room. States are represented as bounded intervals, and participate in ordering relations with events in the tree. It is clear that in many cases, a state should persist throughout the interval spanning subsequent events. This is not captured by the current tree representation. Opposition structures of predicates and gating operations over properties can be expressed as constraints introduced by events, however, but at this stage of development, we have been interested in capturing a coarser temporal ordering representation, very robustly. We believe, however, that annotation using the minimal inclusion relation will allow us to reason about persistence heuristically in the future.

\section{Prerequisites}

Prior work on temporal information extraction has been fairly extensive and is covered in (Mani et al. 2004). Recent research has developed the TimeML annotation scheme (Pustejovsky et al. 2002) (Pustejovsky et al. 2004), as well as a corpus of TimeML-annotated news stories (TimeBank 2004) and annotation tools that go along with it, such as the TANGO tool (Pustejovsky et al. 2003). TimeML flags tensed verbs, adjectives, and nominals that correspond to events and states, tagging instances of them with standard TimeML attributes, including the class of event (perception, reporting, aspectual, state, etc.), tense (past, present, future), grammatical aspect (perfective, progressive, or both), whether it is negated, any modal operators which govern it, and its cardinality if the event occurs more than once. Likewise, time expressions are flagged, and their values normalized, so that Thursday in He left on Thursday would get a resolved ISO time value depending on context (TIMEX2 2004). Finally, temporal relations between events and time expressions (e.g., that the leaving occurs during Thursday) are recorded by means of temporal links (TLINKs) that express Allen-style interval relations (Allen 1984).

Several automatic tools have been developed in conjunction with TimeML, including event taggers (Pustejovsky et al. 2003), time expression taggers (Mani and Wilson 2000), and an exploratory link extractor (Mani et al. 2003).
Temporal reasoning algorithms have also been developed, that apply transitivity axioms to expand the links using temporal closure algorithms (Setzer and Gaizauskas 2001), (Pustejovsky et al. 2003).

However, TimeML is inadequate as a temporal model of discourse: it constructs no global representation of the narrative structure, instead annotating a complex graph that links primitive events and times.

\section{Related Frameworks}

Since the relations in TDMs involve temporal inclusion and temporal ordering, the mentioned events can naturally be mapped to other discourse representations used in computational linguistics. A TDM tree can be converted to a first-order temporal logic representation (where temporal ordering and inclusion operators are added) by expanding the properties of the nodes. These properties include any additional predications made explicitly about the event, e.g., information from thematic arguments and adjuncts. In other words, a full predicate argument representation, e.g., as might be found in the PropBank (Kingsbury and Palmer 2002), can be associated with each node.

TDMs can also be mapped to Discourse Representation Structures (DRS) (which in turn can be mapped to a logical form). Since TDMs represent events as pairs of time points (which can be viewed as intervals), and DRT represents events as primitives, we can reintroduce time intervals based on the standard DRT approach $(\mathrm{e} \subseteq \mathrm{t}$ for events, e $\mathrm{O} t$ for states, except for present tense states, where $\mathrm{t} \subseteq \mathrm{e}$ ).

Consider an example from the Discourse Representation Theory (DRT) literature (from Kamp and Reyle 1993):

(3) a. A man entered the White Hart. b. He

was wearing a black jacket. c. Bill served

him a beer.

The TDM is $<\mathrm{T} 3, \mathrm{C} 3>$ below, with internal properties of the nodes as shown:

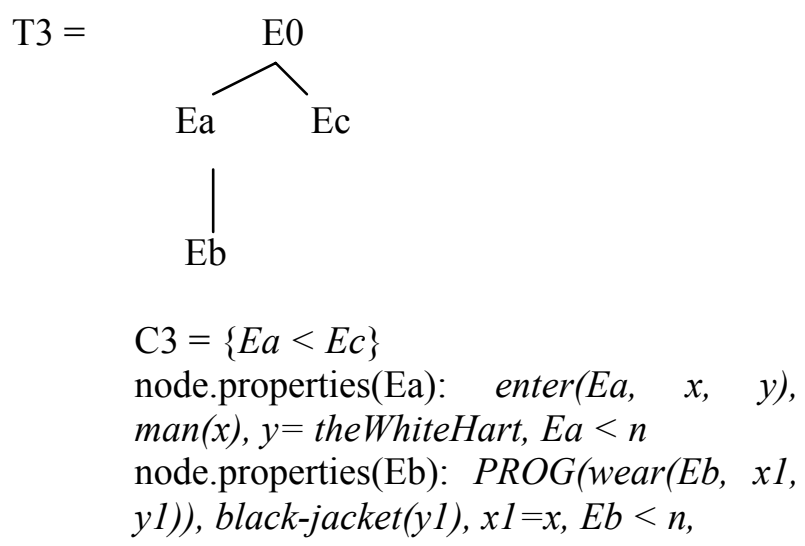


node.properties(Ec): serve(Ec, x2, y2, z), beer(z), $\mathrm{x} 2=$ Bill, $\quad \mathrm{y} 2=\mathrm{x}, \quad \mathrm{Ec}<\mathrm{n}$ From T3: $\mathrm{Eb} \subseteq \mathrm{Ea}$

From C3: $E a<E c$

The DRT representation is shown below (here we have created variables for the reference times):

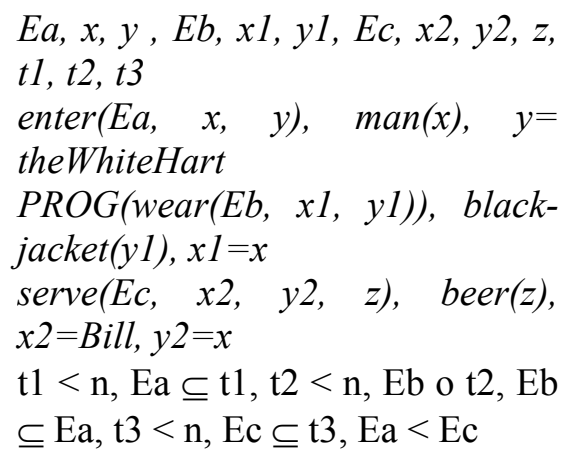

Note that we are by no means claiming that DRSs and TDMs are equivalent. TDMs are tree-structured and DRSs are not, and the inclusion relations involving our abstract events, i.e., Ea $\subseteq$ $\mathrm{E} 0$ and $\mathrm{Ec} \subseteq \mathrm{E} 0$, are not usually represented in DRT. Nevertheless, there are many similarities between TDMs and DRT which are worth examining for semantic and computational properties. Furthermore, SDRT (Asher and Lascarides 2003) extends DRT to include discourse relations. SDRT and RST both differ fundamentally from TDMs, since we dispense with rhetorical relations.

It should be pointed out, nevertheless, that TDMs, as modeled so far, do not represent modality and intensional contexts in the tree structure. (However, information about modality and negation is stored in the nodes based on TimeML preprocessing). One way of addressing this issue is to handle lexically derived modal subordination (such as believe and want) by introducing embedded events, linked to the modal predicate by subordinating relations. For example, in the sentence John believed that Mary graduated from Harvard, the complement event is represented as a subtree linked by a lexical relation.

DLTAG (Webber et al. 2004) is a model of discourse structure where explicit or implicit discourse markers relating only primitive discourse units. Unlike TDMs, where the nodes in the tree can contain embedded structures, DLTAG is a local model of discourse structure; it thus provides a set of binary relations, rather than a tree Like TDMs, however, DLTAG models discourse structure without postulating the existence of rhetorical relations in the discourse tree. Instead, the rhetorical relations appear as predicates in the semantic forms for discourse markers. In this respect, they differ from TDMs, which do not commit to specific rhetorical relations.

Spejewski (1994) developed a tree-based model of the temporal structure of a sequence of sentences. Her approach is based on relations of temporal coordination and subordination, and is thus a major motivation for our own approach. However, her approach mixes both reference times and events in the same representation, so that the parent-child relation sometimes represents temporal anchoring, and at other times coordination. In the above example of John walked home. He was feeling great, her approach would represent the "reference time" of the state (of feeling great) as being part of the event of walking as well as part of the state, resulting in a graph rather than a strict tree. Note that our approach uses minimality.

(Hitzeman et al. 1995) developed a computational approach to distinguish various temporal threads in discourse. The idea here, based on the notion of temporal centering, is that there is one 'thread' that the discourse is currently following. Thus, in (1) above, each utterance is associated with exactly one of two threads: (i) going into the florist's shop and (ii) interacting with Mary. Hitzeman et al. prefer an utterance to continue a current thread which has the same tense or is semantically related to it, so that in (1) above, utterance $d$ would continue the thread (i) above based on tense. In place of world knowledge, however, semantic distance between utterances is used, presumably based on lexical relationships. Whether such semantic similarity is effective is a matter for evaluation, which is not discussed in their paper. For example, it isn't clear what would rule out (1c) as continuing thread (i).

While TDMs do not commit to rhetorical relations, our expectation is that they can be used as an intermediate representation for rhetorical parsing. Thus, when event A in a TDM temporally precedes its right sibling $\mathrm{B}$, the rhetorical relation of Narration will typically be inferred. When B precedes is left sibling A, then Explanation will typically be inferred. When A temporally includes a child node $B$, then Elaboration is typically inferred, etc. TDMs are thus a useful shallow representation that can be a useful first step in deriving rhetorical relations; indeed, rhetorical relations may be implicit in the human annotation of such relations, e.g., when explicit discourse markers like "because" indicate a particular temporal order.

\section{Annotation Scheme}


The annotation scheme involves taking each document that has been preprocessed with time expressions and event tags (complying with TimeML) and then representing TDM parse trees and temporal ordering constraints (the latter also compliant with TimeML TLINKS).

Each discourse begins with a root abstract node. As an annotation convention, (A1) in the absence of any overt or covert discourse markers or temporal adverbials, a tense shift will license the creation of an abstract node, with the event with the shifted tense being the leftmost daughter of the abstract node. The abstract node will then be inserted as the child of the immediately preceding text node. In addition, convention (A2) states that in the absence of temporal adverbials and overt or covert discourse markers, a stative event will always be placed as a child of the immediately preceding text event when the latter is non-stative. Further, convention (A3) states that when the previous event is stative, in the absence of temporal adverbials and explicit or implicit discourse markers, the stative event is a sibling of the previous stative (as in a scene-setting fragment of discourse).

We expect that inter-annotator reliability on TDM trees will be quite high, given the transparent nature of the tree structure along with clear annotation conventions. The Appendices provide examples of annotation, to illustrate the simplicity of the scheme as well as potential problems.

\section{Corpora}

We have begun annotating three corpora with Temporal Discourse Model information. The first is the Remedia corpus (remedia.com). There are 115 documents in total, grouped into four reading levels, all of which have been tagged by a human for time expressions in a separate project by Lisa Ferro at MITRE. Each document is short, about 237 words on average, and has a small number of questions after it for reading comprehension.

The Brandeis Reading Corpus is a collection of 100 K-8 Reading Comprehension articles, mined from the web and categorized by level of comprehension difficulty. Articles range from 50-350 words in length. Complexity of the reading task is defined in terms of five basic classes of reading difficulty.

The last is the Canadian Broadcasting Corporation (cbc4kids.ca). The materials are current-event stories aimed at an audience of 8year-old to 13-year-old students. The stories are short (average length around 450 words). More than a thousand articles are available. The
CBC4Kids corpus is already annotated with POS and parse tree markup.

\section{Conclusion}

Our assumption so far has been that the temporal structure of narratives is tree-structured and context-free. Whether the context-free property is violated or not remains to be seen.

Once the annotation effort is completed, we plan to use the annotated corpora in statistical parsing algorithms to construct TDMs. This should allow features from the corpus to be leveraged together to make inferences about narrative structure. While such knowledge source combination is not by any means guaranteed to substitute for commonsense knowledge, it at least allows for the introduction of generic, machine learning methods for extracting narrative structure from stories in any domain. Earlier work in a noncorpus based (Hitzeman et al. 1995) as well as corpus-based setting (Mani et al. 2003) attests to the usefulness of combining knowledge sources for inferring temporal relations. We expect to leverage similar methods in TDM parsing.

We believe that the temporal aspect of discourse provides a handle for investigating discourse structure, thereby simplifying the problem of discourse structure annotation. It is therefore of considerable theoretical interest. Further, being able to understand the structure of narratives will in turn allow us to summarize them and answer temporal questions about narrative structure.

\section{References}

J. F. Allen. 1984. Towards a General Theory of Action and Time. Artificial Intelligence 23: 123-154.

N. Asher and A. Lascarides. 2003. Logics of Conversation. Cambridge University Press.

A. Bell. 1999. News Stories as Narratives. In A. Jaworski and N. Coupland, The Discourse Reader, Routledge, London and New York, 236251.

L. Carlson, D. Marcu and M. E. Okurowski. 2001. Building a discourse-tagged corpus in the framework of rhetorical structure theory. In Proceedings of the 2nd SIGDIAL Workshop on Discourse and Dialogue, Eurospeech 2001, Aalborg, Denmark.

B. Grosz, A. Joshi and S. Weinstein. 1995. Centering: A Framework for Modeling the Local Coherence of Discourse. Computational Linguistics 2(21), pp. 203-225 
J. Hitzeman, M. Moens and C. Grover. 1995. Algorithms for Analyzing the Temporal Structure of Discourse. In Proceedings of the Annual Meeting of the European Chapter of the Association for Computational Linguistics, Utrecht, Netherlands, 1995, 253-260.

J. Hobbs. 1985. On the Coherence and Structure of Discourse. Report No. CSLI-85-37. Stanford, California: Center for the Study of Language and Information, Stanford University.

E. Hovy. 1990. Parsimonious and Profligate Approaches to the Question of Discourse Structure Relations. In Proceedings of the Fifth International Workshop on Natural Language Generation.

H. Kamp and U. Reyle. 1993. Tense and Aspect. Part 2, Chapter 5 of From Discourse to Logic, 483-546.

P. Kingsbury and M. Palmer. 2002. From Treebank to PropBank. In Proceedings of the 3rd International Conference on Language Resources and Evaluation (LREC-2002), Las Palmas, Spain.

I.. Mani, B. Schiffman and J. Zhang. 2003. Inferring Temporal Ordering of Events in News. Proceedings of the Human Language Technology Conference, HLT'03.

I. Mani and G. Wilson. 2000. Processing of News. Proceedings of the 38th Annual Meeting of the Association for Computational Linguistics (ACL'2000), 69-76.

I. Mani, J. Pustejovsky and R. Gaizauskas. 2004. The Language of Time: A Reader. Oxford University Press, to appear.

W. Mann and S. Thompson. 1988. Rhetorical structure theory: Toward a functional theory of text organization. Text, 8(3): 243-281.

D. Marcu. 2000. The Theory and Practice of Discourse Parsing and Summarization. The MIT Press.

D. Marcu, E. Amorrortu and M. Romera. 1999. Experiments in constructing a corpus of discourse trees. In Proceedings of the $A C L$ Workshop on Standards and Tools for Discourse Tagging, College Park, MD, 48-57.

J. McCarthy and P. Hayes. 1969. Some philosophical problems from the standpoint of artificial intelligence. In B.Meltzer and D. Michie, Eds. Machine Intelligence 4.

J. Pustejovsky, B. Ingria, R. Sauri, J. Castano, J. Littman, R. Gaizauskas, A. Setzer, G. Katz and
I. Mani. 2004. The Specification Language TimeML. In I. Mani, J. Pustejovsky and R. Gaizauskas. The Language of Time: A Reader. Oxford University Press, to appear.

A. Setzer and R. Gaizauskas. 2001. A Pilot Study on Annotating Temporal Relations in Text. ACL 2001, Workshop on Temporal and Spatial Information Processing

B. Spejewski. 1994. Temporal Subordination in Discourse. .Ph.D. Thesis, University of Rochester.

J. Pustejovsky, I. Mani, L. Belanger, B. Boguraev, B. Knippen, J. Littman, A. Rumshisky, A. See, S. Symonenko, J. Van Guilder, L. Van Guilder, M. Verhagen, R. Ingria. 2003. TANGO Final Report. timeml.org.

J. Pustejovsky, L. Belanger, J. Castano, R. Gaizauskas, P. Hanks, R. Ingria, G. Katz, D. Radev, A. Rumshisky, A. Sanfilippo, R. Sauri, B. Sundheim, M. Verhagen. 2002. TERQAS Final Report. timeml.org.

TIMEBANK. 2004. timeml.org.

TIMEX2. 2004. timex2.mitre.org.

B. Webber. 1998. Tense as Discourse Anaphor. Computational Linguistics 14(2): 61-73.

B. Webber, M. Stone, A. Joshi and A. Knott. 2003. Computational Linguistics, 29:4, 545-588.

Appendix A: Examples from (Hitzeman et al. 1995)

1. (a) John entered the room. (b) Mary stood up.

$\mathrm{Ea}$ is inserted as left daughter of root. $\mathrm{Eb}$ is attached as sister (an analogue of a Narration default rhetorical relation).
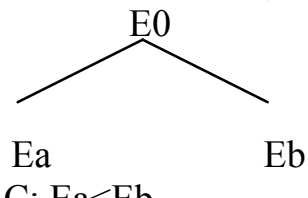

$\mathrm{C}: \mathrm{Ea}<\mathrm{Eb}$

2. (a) John entered the room. (b) Mary was seated behind the desk.

$\mathrm{Ea}$ is anchored as left daughter of root. $\mathrm{Eb}$ is a tensed stative, and is embedded below Ea.

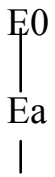



$\mathrm{Eb}$
$\mathrm{C}: \mathrm{Eb} \subseteq_{\min } \mathrm{Ea}$

3. (a) John fell. (b) Mary pushed him.

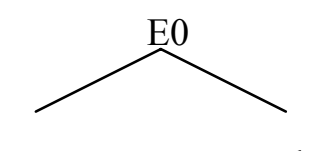

$\mathrm{Ea} \quad \mathrm{Eb}$

$\mathrm{C}: \mathrm{Eb}<\mathrm{Ea}$

4. (a) John entered the room because (b) Mary stood up.

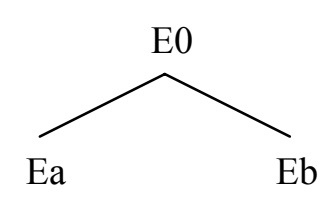

$\mathrm{C}: \mathrm{Eb}<\mathrm{Ea}$

This is due to the "because"-inversion rule.

5. (a) Mary was tired. (b) She was exhausted.

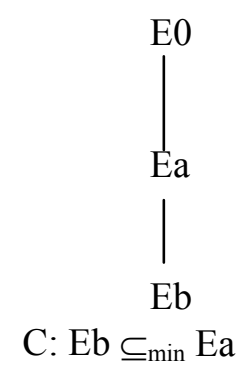

This case, unlike (2), would be an analogue of an Elaboration relation. Here, other knowledge sources, such as a centering (Grosz et al. 1995) could play a role in inferring such a discourse relation.

6. (a) Sam rang the bell. (b) He had lost the key. (c) It had fallen through a hole in his pocket.

$\mathrm{Ea}$ is attached as right branching event. $\mathrm{Eb}$ is attached as sister with precedence constraint relative to Ea coming from the past perfect marking. Ec is attached as sister with precedence constraint relative to $\mathrm{Eb}$ coming from past perfect. Losing the key is explained by (or elaborated by) the description of the key falling through the hole. Hence, it should be an embedding relation on this reading. Nevertheless, the current parse is arguably correct since the falling caused the loss of the key.

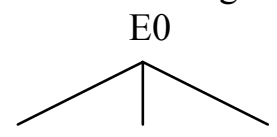

Ea $\left.\left.\right|_{\mathrm{Eb}} ^{\mathrm{E} 1}\right|^{\mathrm{E} 2}$

\section{$\mathrm{C}: \mathrm{Eb}<\mathrm{Ea}, \mathrm{Ec}<\mathrm{Eb}$}

7. (a) John got to work late. (b) He had left the house at 8. (c) $\mathrm{He}$ had eaten a big breakfast.

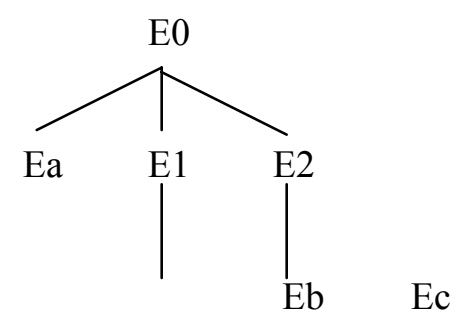

$\mathrm{C}: \mathrm{Eb}<\mathrm{Ea}, \mathrm{Ec}<\mathrm{Eb}$

\section{Appendix B: Level 200 Story from the Brandeis Reading Corpus}

a. David wants to buy a Christmas present for a very special person, his mother.

b. David's father gives him $\$ 5.00$ a week pocket money and

c. David puts $\$ 2.00$ a week into his bank account.

d. After three months David takes $\$ 20.00$ out of his bank account and

e. goes to the shopping mall.

f. He looks and looks for a perfect gift.

g. Suddenly he sees a beautiful brooch in the shape of his favorite pet.

h. He says to himself

i. "Mother loves jewelry, and

j. the brooch costs only $\$ 17.00 . "$

$\mathrm{k}$. He buys the brooch and

1. takes it home.

$\mathrm{m}$. He wraps the present in Christmas paper and

n. places it under the tree.

o. He is very excited and

p. he is looking forward to Christmas morning to see the joy on his mother's face.

q. But when his mother opens the present

r. she screams with fright because s. she sees a spider.

$\mathrm{Ea}, \mathrm{Eb}$, and $\mathrm{Ec}$ are all statively interpreted due to the presence of modification by frequency adverbial TIMEX3 expressions (from TimeML), giving rise to habitual event interpretations. They are embedded inside an abstract E0 node. 


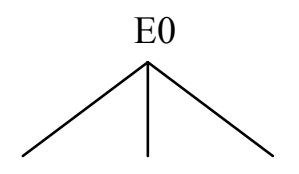

$\mathrm{Ea} \quad \mathrm{Eb} \quad \mathrm{Ec}$

$\mathrm{C}: \mathrm{Ea} \subseteq_{\min } \mathrm{E} 0, \mathrm{~Eb} \subseteq_{\min } \mathrm{E} 0, \mathrm{Ec} \subseteq_{\min } \mathrm{E} 0$

E1 is created with the recognition of the time expression "after three months". Ed is attached as the left daughter node in E1. Ee is attached as sister (default Narrative). Similarly for Ef, Eg, and Eh.

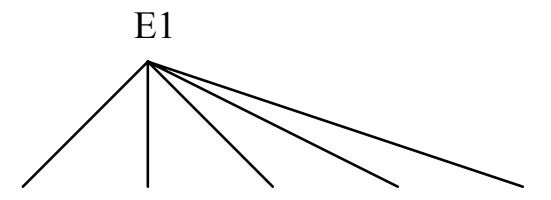

Ed Ee Ef Eg Eh

\section{$\mathrm{C}: \mathrm{Ed}<\mathrm{Ee}, \mathrm{Ee}<\mathrm{Ef}, \mathrm{Ef}<\mathrm{Eg}, \mathrm{Eg}<\mathrm{Eh}$}

The syntactically embedded sentences in (i) and (j) are recognized as states and are embedded within Eh.

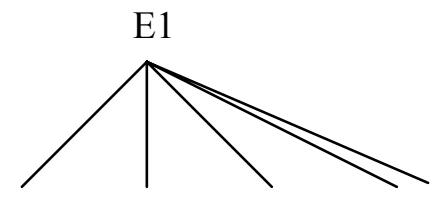

Ed Ee Ef Eg Eh

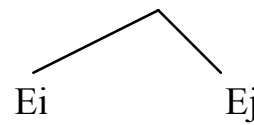

$\mathrm{C}: \mathrm{Ed}<\mathrm{Ee}, \mathrm{Ee}<\mathrm{Ef}, \mathrm{Ef}<\mathrm{Eg}, \mathrm{Eg}<\mathrm{Eh}, \mathrm{Ei}$ in $\mathrm{Eh}, \mathrm{Ej}$ in Eh

Attachment and narrative order holds for Ek, El, Em, and En. The states in Eo and Ep will be embedded under En:

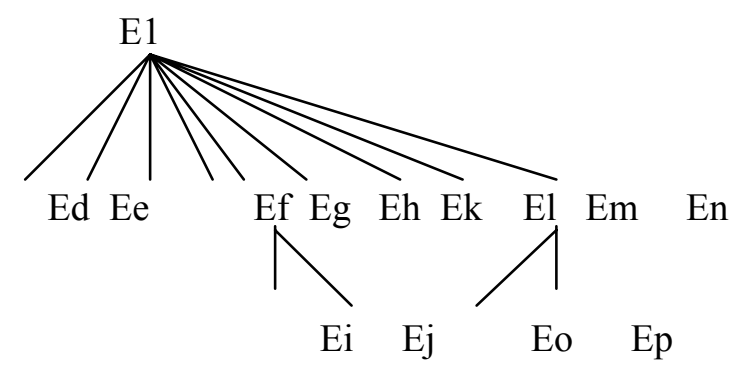

The presence of "when" as a TimeML signal creates a new abstract event, E3, and the subsequent ordering relation $\mathrm{E} 3>\mathrm{E} 2$.

Finally, narration continues under E3 with Eq, $\mathrm{Er}$, and Es, as daughters to E3, with the additional constraint of "because-inversion", Es $<$ Er.

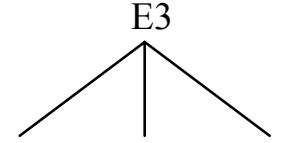

Eq Er Es

$\mathrm{C}: \mathrm{Eq}<\mathrm{Er}, \mathrm{Es}<\mathrm{Er}, \mathrm{Eq}<\mathrm{Es}$

The TDM for the entire article is represented below:

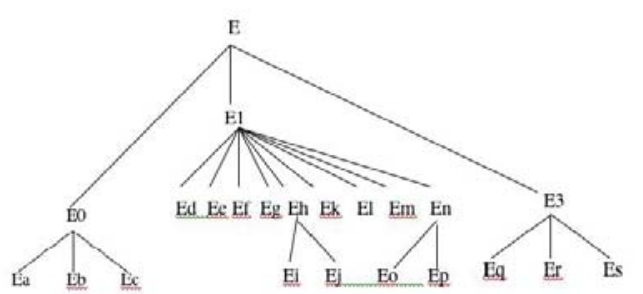

